PROCEEDINGS OF THE

AMERICAN MATHEMATICAL SOCIETY

Volume 136, Number 7, July 2008, Pages 2303-2312

S 0002-9939(08)09199-5

Article electronically published on February 28, 2008

\title{
DETECTING COMPLETENESS FROM EXT-VANISHING
}

\author{
ANDERS J. FRANKILD AND SEAN SATHER-WAGSTAFF \\ (Communicated by Bernd Ulrich) \\ Dedicated to Lex Remington
}

\begin{abstract}
Motivated by work of C. U. Jensen, R.-O. Buchweitz, and H. Flenner, we prove the following result. Let $R$ be a commutative noetherian ring and $\mathfrak{a}$ an ideal in the Jacobson radical of $R$. Let $\widehat{\mathfrak{R a}}$ be the a-adic completion of $R$. If $M$ is a finitely generated $R$-module such that $\operatorname{Ext}_{R}^{i}(M)=0$ for all $i \neq 0$, then $M$ is a-adically complete.
\end{abstract}

\section{INTRODUCTION}

A result of Jensen [13, (8.1)] characterizes the completeness property of a semilocal ring in terms of Ext-vanishing: If $R$ is a commutative noetherian ring, then it is a finite product of complete local rings if and only if $\operatorname{Ext}_{R}^{i}(B, M)=0$ for $i \neq 0$ whenever $B$ is flat and $M$ is finitely generated over $R$. In their investigation of Hochschild homology, Buchweitz and Flenner [3, (2.3)] recover one implication of the local case of this result: Let $R$ be a ring and $\mathfrak{m} \subset R$ a maximal ideal; if $M$ is an $\mathfrak{m}$-adically complete $R$-module, then $\operatorname{Ext}_{R}^{i}(B, M)=0$ for all $i \neq 0$ and each flat $R$-module $B$; see also [8, (3.7)] for the local case.

In this paper, we investigate converses to the Buchweitz-Flenner result: If $M$ is an $R$-module such that $\operatorname{Ext}_{R}^{i}(B, M)=0$ for all $i \neq 0$ and each flat $R$-module $B$, must $M$ be $\mathfrak{m}$-adically complete? One readily sees that this need not be the case when $M$ is not finitely generated. If $R$ is a local domain with $\operatorname{dim}(R)>0$ and $M$ is the quotient field of $R$, then $M$ is not $\mathfrak{m}$-adically complete. However, $M$ is injective so $\operatorname{Ext}_{R}^{i}(B, M)=0$ for all $i \neq 0$ and each $R$-module $B$.

The following result is proved in 3.1 . When $M$ finitely generated, it shows that the completeness of $M$ can be ascertained from the vanishing of the Ext-modules against a single flat module, namely $\widehat{R}$.

Theorem A. Let $R$ be a commutative noetherian ring and $\mathfrak{a}$ an ideal in the Jacobson radical of $R$. Let $\widehat{R}^{\mathfrak{a}}$ be the $\mathfrak{a}$-adic completion of $R$ and let $M$ be a finitely generated $R$-module. The following conditions are equivalent:

(i) $M$ is a-adically complete.

(ii) $\operatorname{Ext}_{R}^{i}\left(\widehat{R}^{\mathfrak{a}}, M\right)=0$ for all $i \neq 0$.

Received by the editors June 28, 2006, and, in revised form, March 28, 2007.

2000 Mathematics Subject Classification. Primary 13B35, 13D07, 13D25, 13D45, 13 J10.

Key words and phrases. Completions, completeness, ext, local cohomology, local homology.

This research was conducted while the first author had a Steno Stipend from the Danish Research Council.

(C)2008 American Mathematical Society Reverts to public domain 28 years from publication 
(iii) $\operatorname{Ext}_{R}^{i}\left(\widehat{R}^{\mathfrak{a}}, M\right)=0$ for all $i=1, \ldots, \operatorname{dim}_{R}(M)$.

As a consequence of this theorem we obtain the following two results. The first is proved in 3.3. and the second is contained in Corollary 3.9.

Theorem B. The ring $R$ is $\mathfrak{a}$-adically complete if and only if the completion $\widehat{R}^{\mathfrak{a}}$ is module-finite over $R$.

Theorem C. Let $M, N$ be finitely generated $R$-modules and $t$ an integer such that $\operatorname{Ext}_{R}^{i}(N, M)=0$ for each $i<t$. If $\operatorname{Ext}_{R}^{i}\left(\widehat{N}^{\mathfrak{a}}, M\right)=0$ for each $i \neq t$, then $\operatorname{Ext}_{R}^{i}(N, M)=0$ for each $i \neq t$ and $\operatorname{Ext}_{R}^{t}(N, M)$ is a-adically complete.

To prove these results, we employ a combination of classical module-theory and derived category techniques. Preliminary module-theoretic results are presented in Section 1. Requisite derived category notions are discussed in Section 2.

\section{Analytic conductor submodules}

Throughout this work, $R$ is a commutative noetherian ring and $\mathfrak{a}$ is an ideal contained in the Jacobson radical of $R$.

Lemma 1.1. If $M$ is a finitely generated $R$-module, then $M$ admits a unique maximal $\mathfrak{a}$-adically complete submodule $\mathrm{C}_{M}^{\mathfrak{a}}$.

Proof. Let $\mathbf{C}^{\mathfrak{a}}(M)$ denote the collection of $\mathfrak{a}$-adically complete submodules of $M$ which is nonempty because it contains the zero submodule. Since $M$ is noetherian, this collection contains maximal elements, each of which is finitely generated. Let $N, N^{\prime} \in \mathbf{C}^{\mathfrak{a}}(M)$ be maximal elements and suppose that $N \neq N^{\prime}$. By maximality, one has $N \nsubseteq N^{\prime}$ and so $N \subsetneq N+N^{\prime}$. In particular, $N+N^{\prime}$ is not a-adically complete. However, the module $N \oplus N^{\prime}$ is finitely generated and a-adically complete. Hence, the homomorphic image $N+N^{\prime}$ of $N \oplus N^{\prime}$ is a-adically complete, a contradiction. Thus, $N=N^{\prime}$, and the maximal element of $\mathbf{C}^{\mathfrak{a}}(M)$ is unique.

The submodule $\mathrm{C}_{M}^{\mathfrak{a}}$ is the analytic conductor of $M$ with respect to $\mathfrak{a}$. It is the largest $R$-submodule of $M$ that is also an $\widehat{R}^{\mathfrak{a}}$-module. Before presenting an important property of $\mathrm{C}_{M}^{\mathfrak{a}}$ for this work, we introduce some frequently used maps.

1.2. Let $M$ be an $R$-module. The map $g_{M}^{\mathfrak{a}}: \operatorname{Hom}_{R}\left(\widehat{R}^{\mathfrak{a}}, M\right) \rightarrow M$ is given by $g_{M}^{\mathfrak{a}}(\varphi)=\varphi(1)$, and $\varepsilon_{M}^{\mathfrak{a}}: M \rightarrow \widehat{M}^{\mathfrak{a}}$ is the natural inclusion. Assume now that $M$ is finitely generated, so that $\mathrm{C}_{M}^{\mathfrak{a}}$ is defined. Let $i_{M}^{\mathfrak{a}}: \mathrm{C}_{M}^{\mathfrak{a}} \rightarrow M$ denote the natural inclusion. The map $f_{M}^{\mathfrak{a}}: \mathrm{C}_{M}^{\mathfrak{a}} \rightarrow \operatorname{Hom}_{R}\left(\widehat{R}^{\mathfrak{a}}, M\right)$ is given by $f_{M}^{\mathfrak{a}}(m)(r)=r m$.

The next result yields a well-defined map $k_{M}^{\mathfrak{a}}: \operatorname{Hom}_{R}\left(\widehat{R}^{\mathfrak{a}}, M\right) \rightarrow \mathrm{C}_{M}^{\mathfrak{a}}$, given by $k_{M}^{\mathfrak{a}}(\varphi)=\varphi(1)$, such that $g_{M}^{\mathfrak{a}}=i_{M}^{\mathfrak{a}} k_{M}^{\mathfrak{a}}$.

Lemma 1.3. If $M$ is a finitely generated $R$-module, then the natural inclusion $\operatorname{Hom}_{R}\left(\widehat{R}^{\mathfrak{a}}, i_{M}^{\mathfrak{a}}\right): \operatorname{Hom}_{R}\left(\widehat{R}^{\mathfrak{a}}, \mathrm{C}_{M}^{\mathfrak{a}}\right) \rightarrow \operatorname{Hom}_{R}\left(\widehat{R}^{\mathfrak{a}}, M\right)$ is bijective.

Proof. By left-exactness of $\operatorname{Hom}_{R}\left(\widehat{R}^{\mathfrak{a}},-\right)$ the given map is injective. To see that this map is surjective, fix $\varphi \in \operatorname{Hom}_{R}\left(\widehat{R}^{\mathfrak{a}}, M\right)$; it suffices to $\operatorname{show} \operatorname{Im}(\varphi) \subseteq \mathrm{C}_{M}^{\mathfrak{a}}$. The image $\operatorname{Im}(\varphi)$ is finitely generated over $R$ and a homomorphic image of the $\mathfrak{a}$-adically complete $R$-module $\widehat{R}^{\mathfrak{a}}$. Hence, $\operatorname{Im}(\varphi)$ is a-adically complete, and the desired conclusion follows from Lemma 1.1. 


\section{Derived local homology and COHOMOlogy}

We work in the derived category $\mathrm{D}(R)$ of complexes of $R$-modules, indexed homologically. References on the subject include [9, 11]. A complex $X$ is homologically bounded to the right if $\mathrm{H}_{i}(X)=0$ for all $i \ll 0$; it is homologically degreewise finite if $\mathrm{H}_{i}(X)$ is finitely generated for each $i$; it is homologically finite if $\bigoplus_{i} \mathrm{H}_{i}(X)$ is finitely generated; and it is homologically concentrated in degree $s$ if $\mathrm{H}_{i}(X)=0$ for all $i \neq s$. Isomorphisms in $\mathrm{D}(R)$ are identified by the symbol $\simeq$, as are quasiisomorphisms in the category of complexes. For $X, Y \in \mathrm{D}(R) \operatorname{set} \inf (X)$ and $\sup (X)$ to be the infimum and supremum, respectively, of the set $\left\{n \in \mathbf{Z} \mid \mathrm{H}_{n}(X) \neq 0\right\}$. Let $X \otimes{ }_{R}^{\mathrm{L}} Y$ and $\mathbf{R H o m}_{R}(X, Y)$ denote the left-derived tensor product and right-derived homomorphism complexes, respectively.

The left-derived local homology and right-derived local cohomology functors with support in an ideal $\mathfrak{a}$ are denote $\mathbf{L} \Lambda^{\mathfrak{a}}(-)$ and $\mathbf{R} \Gamma_{\mathfrak{a}}(-)$, respectively; see [1, 10]. These are computed as follows. If $P \stackrel{\simeq}{\longrightarrow} X \stackrel{\simeq}{\longrightarrow} J$ are K-projective and K-injective resolutions, respectively, as in [2, 16], then

$$
\begin{aligned}
\Lambda^{\mathfrak{a}}(-) & =\lim _{n}\left(R / \mathfrak{a}^{n} \otimes_{R}-\right), & \Gamma_{\mathfrak{a}}(-) & =\operatorname{colim}_{n} \operatorname{Hom}_{R}\left(R / \mathfrak{a}^{n},-\right), \\
\mathbf{L} \Lambda^{\mathfrak{a}}(X) & =\Lambda^{\mathfrak{a}}(P), & \mathbf{R} \Gamma_{\mathfrak{a}}(X) & =\Gamma_{\mathfrak{a}}(J) .
\end{aligned}
$$

Note that the functor $\Gamma_{\mathfrak{a}}(-)$ is left-exact while $\Lambda^{\mathfrak{a}}(-)$ is neither left- nor right-exact.

2.1. Here is a catalog of properties of $\mathbf{L} \Lambda^{\mathfrak{a}}(-)$ and $\mathbf{R} \Gamma_{\mathfrak{a}}(-)$ that we will utilize.

(a) There are natural transformations of functors on $\mathrm{D}(R)$ [1, $\left.(0.3)^{*}\right]$,

$$
\mathbf{R} \Gamma_{\mathfrak{a}}(-) \stackrel{\gamma}{\rightarrow} 1_{\mathrm{D}(R)}(-) \stackrel{\nu}{\rightarrow} \mathbf{L} \Lambda^{\mathfrak{a}}(-) .
$$

(b) The following are equivalences of functors on $\mathrm{D}(R)$ [1, Cor. to $\left.(0.3)^{*}\right]$ :

$$
\mathbf{L} \Lambda^{\mathfrak{a}}\left(\mathbf{R} \Gamma_{\mathfrak{a}}(-)\right) \stackrel{\mathbf{L} \Lambda^{\mathfrak{a}}(\gamma)}{\longrightarrow} \mathbf{L} \Lambda^{\mathfrak{a}}(-) \quad \text { and } \quad \mathbf{R} \Gamma_{\mathfrak{a}}(-) \stackrel{\mathbf{R} \Gamma_{\mathfrak{a}}(\nu)}{\longrightarrow} \mathbf{R} \Gamma_{\mathfrak{a}}\left(\mathbf{L} \Lambda^{\mathfrak{a}}(-)\right) .
$$

(c) One has natural equivalences of functors on $\mathrm{D}(R)$ ([1, (0.3)] and [14, (3.1.2)]):

$$
\mathbf{L} \Lambda^{\mathfrak{a}}(-) \simeq \mathbf{R} \operatorname{Hom}_{R}\left(\mathbf{R} \Gamma_{\mathfrak{a}}(R),-\right) \quad \text { and } \quad \mathbf{R} \Gamma_{\mathfrak{a}}(-) \simeq \mathbf{R} \Gamma_{\mathfrak{a}}(R) \otimes_{R}^{\mathbf{L}}-.
$$

(d) (Adjointness) There is a natural equivalence of bifunctors on $\mathrm{D}(R)$,

$$
\mathbf{R H o m}_{R}\left(\mathbf{R} \Gamma_{\mathfrak{a}}(-),-\right) \underset{\simeq}{\stackrel{\theta}{\longrightarrow}} \mathbf{R} \operatorname{Hom}_{R}\left(-, \mathbf{L} \Lambda^{\mathfrak{a}}(-)\right),
$$

such that, for all complexes $X$ and $Y$ the next diagram commutes [1, (0.3)].

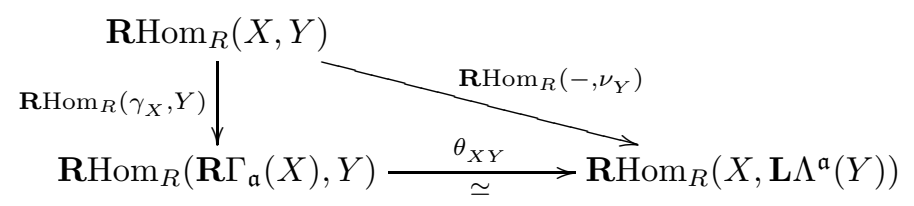

In particular, the morphism $\mathbf{R H o m}{ }_{R}\left(\gamma_{X}, Y\right)$ is an isomorphism in $\mathbf{D}(R)$ if and only if $\mathbf{R H o m}_{R}\left(-, \nu_{Y}\right)$ is so.

(e) One has a natural equivalence of functors on the full subcategory of $\mathrm{D}(R)$ of complexes that are homologically finite and bounded to the right $[8,(2.8)]$,

$$
\mathbf{L} \Lambda^{\mathfrak{a}}(-) \simeq-\bigotimes_{R} \widehat{R}^{\mathfrak{a}}
$$


(f) Parts (므)-(ㄷ) yield equivalences of (bi)functors on $\mathrm{D}(R)$; see, e.g., 4 , (A.4.22)]:

$$
\begin{gathered}
\mathbf{R} \Gamma_{\mathfrak{a}}(R) \otimes_{R}^{\mathbf{L}} \mathbf{L} \Lambda^{\mathfrak{a}}(-) \simeq \mathbf{R} \Gamma_{\mathfrak{a}}(-), \\
\mathbf{L} \Lambda^{\mathfrak{a}}\left(\mathbf{R} \operatorname{Hom}_{R}(-,-)\right) \simeq \mathbf{R} \operatorname{Hom}_{R}\left(-, \mathbf{L} \Lambda^{\mathfrak{a}}(-)\right) .
\end{gathered}
$$

(g) If $X$ is homologically bounded to the right, then it admits a K-projective resolution $P \stackrel{\simeq}{\longrightarrow} X$ such that $X_{i}=0$ for each $i \leqslant \inf (X)$, and so

$$
\inf \left(\mathbf{L} \Lambda^{\mathfrak{a}}(X)\right)=\inf \left(\Lambda^{\mathfrak{a}}(P)\right) \geqslant \inf (P)=\inf (X) .
$$

We now verify facts about $\mathbf{L} \Lambda^{\mathfrak{a}}(-)$ and $\mathbf{R} \Gamma_{\mathfrak{a}}(-)$ for the sequel. Fix $M \in \mathrm{D}(R)$ with K-injective resolution $M \stackrel{\simeq}{\longrightarrow} J$. The map $g_{J}^{\mathfrak{a}}: \operatorname{Hom}_{R}\left(\widehat{R}^{\mathfrak{a}}, J\right) \rightarrow J$ given by $\varphi \mapsto \varphi(1)$ describes a well-defined morphism $h_{M}^{\mathfrak{a}}: \mathbf{R H o m}_{R}\left(\widehat{R}^{\mathfrak{a}}, M\right) \rightarrow M$ in $\mathrm{D}(R)$.

Lemma 2.2. If $M$ is an $R$-complex, then the induced morphisms

$$
\begin{aligned}
& \mathbf{L} \Lambda^{\mathfrak{a}}\left(h_{M}^{\mathfrak{a}}\right): \mathbf{L} \Lambda^{\mathfrak{a}}\left(\mathbf{R H o m}_{R}\left(\widehat{R}^{\mathfrak{a}}, M\right)\right) \rightarrow \mathbf{L} \Lambda^{\mathfrak{a}}(M), \\
& \mathbf{R} \Gamma_{\mathfrak{a}}\left(h_{M}^{\mathfrak{a}}\right): \mathbf{R} \Gamma_{\mathfrak{a}}\left(\mathbf{R H o m}_{R}\left(\widehat{R}^{\mathfrak{a}}, M\right)\right) \rightarrow \mathbf{R} \Gamma_{\mathfrak{a}}(M)
\end{aligned}
$$

are isomorphisms in $\mathrm{D}(R)$. In particular, if $\mathbf{L} \Lambda^{\mathfrak{a}}(M) \neq 0$ or $\mathbf{R} \Gamma_{\mathfrak{a}}(M) \neq 0$, then $\mathbf{R H o m}_{R}\left(\widehat{R}^{\mathfrak{a}}, M\right) \not ぇ 0$.

Proof. For the first isomorphism, it suffices to check that the morphism

$$
\mathbf{R H o m}_{R}\left(\mathbf{R} \Gamma_{\mathfrak{a}}(R), \mathbf{R H o m}_{R}\left(\widehat{R}^{\mathfrak{a}}, M\right)\right) \stackrel{\mathbf{R} \operatorname{Hom}_{R}\left(\mathbf{R} \Gamma_{\mathfrak{a}}(R), h_{M}^{\mathfrak{a}}\right)}{\longrightarrow} \mathbf{R} \operatorname{Hom}_{R}\left(\mathbf{R} \Gamma_{\mathfrak{a}}(R), M\right)
$$

is an isomorphism in $\mathrm{D}(R)$; see 2.1( (C). In the commutative diagram

$$
\begin{aligned}
& \mathbf{R H o m}_{R}\left(\mathbf{R} \Gamma_{\mathfrak{a}}(R), \mathbf{R H o m}_{R}\left(\widehat{R}^{\mathfrak{a}}, M\right)\right) \stackrel{(1)}{\simeq} \mathbf{R H o m}_{R}\left(\mathbf{R} \Gamma_{\mathfrak{a}}(R) \otimes_{R}^{\mathbf{L}} \widehat{R}^{\mathfrak{a}}, M\right) \\
& \mathbf{R} \operatorname{Hom}_{R}\left(\mathbf{R} \Gamma_{\mathfrak{a}}(R), h_{M}^{\mathfrak{a}}\right) \downarrow \quad \downarrow \mathbf{R} \operatorname{Hom}_{R}\left(\mathbf{R} \Gamma_{\mathfrak{a}}(R) \otimes_{R}^{\mathbf{L}} \varepsilon_{R}^{\mathfrak{a}}, M\right)
\end{aligned}
$$

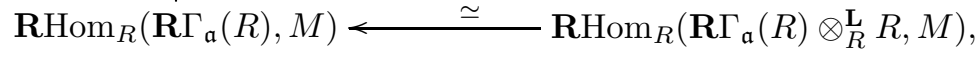

(1) is adjunction and $\varepsilon_{R}^{\mathfrak{a}}: R \rightarrow \widehat{R}^{\mathfrak{a}}$ is the natural inclusion. Since $\mathbf{R} \Gamma_{\mathfrak{a}}(R) \otimes_{R} \varepsilon_{R}^{\mathfrak{a}}$ is an isomorphism by 2.1(f), the same is true of $\mathbf{R} \operatorname{Hom}_{R}\left(\mathbf{R} \Gamma_{\mathfrak{a}}(R) \otimes_{R}^{\mathbf{L}} \varepsilon_{R}^{\mathfrak{a}}, M\right)$. The diagram implies that $\mathbf{R} \operatorname{Hom}_{R}\left(\mathbf{R} \Gamma_{\mathfrak{a}}(R), h_{M}^{\mathfrak{a}}\right)$ is an isomorphism.

For the second isomorphism, use the equivalence of 2.1(b) to see that the vertical maps in the following commutative diagram are isomorphisms:

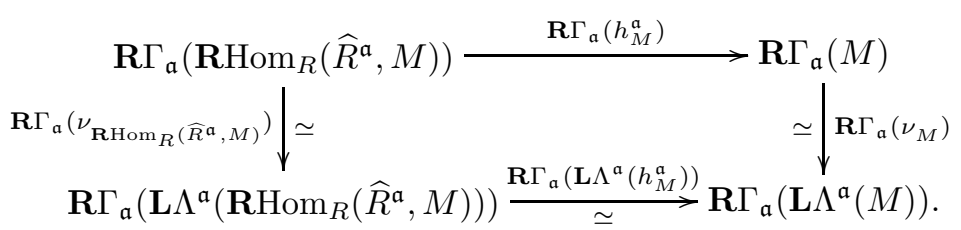

The morphism $\mathbf{R} \Gamma_{\mathfrak{a}}\left(\mathbf{L} \Lambda^{\mathfrak{a}}\left(h_{M}^{\mathfrak{a}}\right)\right)$ is an isomorphism in $\mathrm{D}(R)$ because we have shown that $\mathbf{L} \Lambda^{\mathfrak{a}}\left(h_{M}^{\mathfrak{a}}\right)$ is so. The diagram shows that $\mathbf{R} \Gamma_{\mathfrak{a}}\left(h_{M}^{\mathfrak{a}}\right)$ is an isomorphism as well.

The final statement follows from the additivity of $\mathbf{L} \Lambda^{\mathfrak{a}}(-)$ and $\mathbf{R} \Gamma_{\mathfrak{a}}(-)$.

Lemma 2.3. If $M, N$ are homologically finite $R$-complexes, then the complex $X=$ $\mathbf{R H o m}_{R}(N, M)$ is homologically degreewise finite and $\mathbf{L} \Lambda^{\mathfrak{a}}(X) \simeq X \otimes_{R}^{\mathbf{L}} \widehat{R}^{\mathfrak{a}}$. In particular, one has $\inf \left(\mathbf{L} \Lambda^{\mathfrak{a}}(X)\right)=\inf (X)$ and $\sup \left(\mathbf{L} \Lambda^{\mathfrak{a}}(X)\right)=\sup (X)$. 
Proof. The finiteness of each $\mathrm{H}_{i}(X)$ is standard. A verification of the isomorphism is essentially in [6. Proof of (5.9)]. The flatness of $R \rightarrow \widehat{R}^{\mathfrak{a}}$ implies $\mathrm{H}_{i}\left(\mathbf{L} \Lambda^{\mathfrak{a}}(X)\right) \cong$ $\mathrm{H}_{i}(X) \otimes_{R}^{\mathbf{L}} \widehat{R}^{\mathfrak{a}}$, and the equalities follow from the faithful flatness of $R \rightarrow \widehat{R}^{\mathfrak{a}}$.

We next prove a vanishing result akin to [3, (2.3)]. Note that $M$ is not assumed to be finitely generated.

Proposition 2.4. Let $M$ be an R-module such that the morphism $\nu_{M}: M \rightarrow$ $\mathbf{L} \Lambda^{\mathfrak{a}}(M)$ is an isomorphism in $\mathrm{D}(R)$. Then $\operatorname{Ext}_{R}^{i}\left(\widehat{R}^{\mathfrak{a}}, M\right)=0$ for each $i \neq 0$, and the evaluation map $g_{M}^{\mathfrak{a}}: \operatorname{Hom}_{R}\left(\widehat{R}^{\mathfrak{a}}, M\right) \rightarrow M$ is an isomorphism.

Proof. Because the morphism $\nu_{M}: M \rightarrow \mathbf{L} \Lambda^{\mathfrak{a}}(M)$ is an isomorphism in $\mathrm{D}(R)$, the same is true of $\mathbf{R H o m}_{R}\left(X, \nu_{M}\right): \mathbf{R} \operatorname{Hom}_{R}(X, M) \rightarrow \mathbf{R} \operatorname{Hom}_{R}\left(X, \mathbf{L} \Lambda^{\mathfrak{a}}(M)\right)$ for each $R$-complex $X$. From 2.1(d) it follows that the morphism

$$
\mathbf{R H o m}_{R}\left(\gamma_{X}, M\right): \mathbf{R H o m}_{R}(X, M) \rightarrow \mathbf{R H o m}_{R}\left(\mathbf{R} \Gamma_{\mathfrak{a}}(X), M\right)
$$

is an isomorphism in $\mathrm{D}(R)$.

The naturality of $\gamma$ provides the following commutative diagram in $\mathrm{D}(R)$.

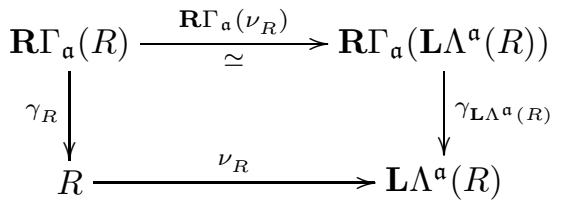

An application of $\mathbf{R H o m}(-, M)$ yields the following commutative diagram in $\mathrm{D}(R)$ :

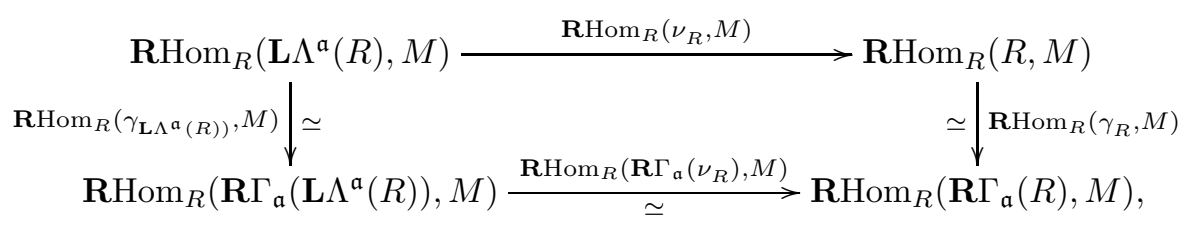

where the vertical morphisms are isomorphisms because of the argument of the previous paragraph. Hence, the morphism $\mathbf{R} \operatorname{Hom}_{R}\left(\nu_{R}, M\right)$ is also an isomorphism.

Next consider the commutative triangle

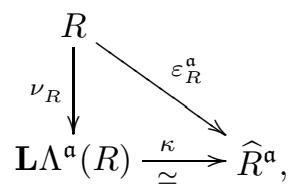

where $\kappa$ is obtained by taking degree 0 homology; see, e.g., 2.1(e). Apply $\mathbf{R H o m}(-, M)$ to produce the following commutative diagram in $\mathrm{D}(R)$ :

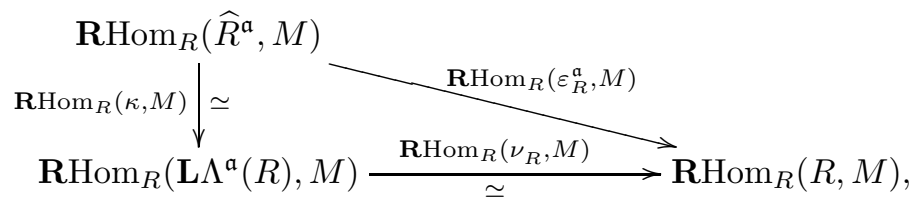

which implies that $\mathbf{R H o m}_{R}\left(\varepsilon_{R}^{\mathfrak{a}}, M\right)$ is an isomorphism in $\mathrm{D}(R)$. 
In the final commutative diagram,

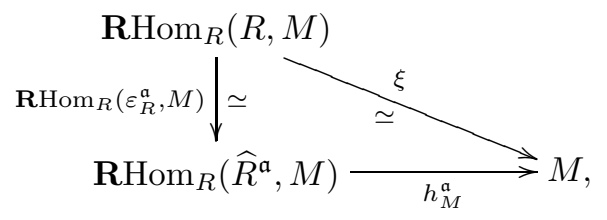

the morphism $\xi$ is the natural evaluation isomorphism. The diagram shows that $h_{M}^{\mathfrak{a}}$ is an isomorphism in $\mathrm{D}(R)$. Since $M$ is a module, this implies $\operatorname{Ext}_{R}^{i}\left(\widehat{R}^{\mathfrak{a}}, M\right)=0$ for each $i \neq 0$ and further that the induced map $\mathrm{H}_{0}\left(h_{M}^{\mathfrak{a}}\right): \operatorname{Hom}_{R}\left(\widehat{R}^{\mathfrak{a}}, M\right) \rightarrow M$ is bijective. The definitions yield an equality $\mathrm{H}_{0}\left(h_{M}^{\mathfrak{a}}\right)=g_{M}^{\mathfrak{a}}$, completing the proof.

Remark 2.5. If $M$ is an $R$-module such that $M \cong \widehat{M}^{\mathfrak{a}}$, then $M \simeq \mathbf{L} \Lambda^{\mathfrak{a}}(M)$. Indeed, the isomorphism $M \cong \widehat{M}^{\mathfrak{a}}$ shows that $M$ is an $\widehat{R}^{\mathfrak{a}}$-module. Let $P$ be an $\widehat{R}^{\mathfrak{a}}$-free resolution of $M$. Then $P$ is an $R$-flat resolution of $M$ consisting of a-adically complete modules. Thus, one has $\mathbf{L} \Lambda^{\mathfrak{a}}(M) \simeq \Lambda^{\mathfrak{a}}(P) \cong P \simeq M$.

We are now in a position to give a useful alternate description of the analytic conductor submodule $\mathrm{C}_{M}^{\mathfrak{a}}$; see 1.2 for the definitions of the maps.

Proposition 2.6. Let $M$ be a finitely generated R-module. The homomorphisms $f_{M}^{\mathfrak{a}}: \mathrm{C}_{M}^{\mathfrak{a}} \rightarrow \operatorname{Hom}_{R}\left(\widehat{R}^{\mathfrak{a}}, M\right)$ and $k_{M}^{\mathfrak{a}}: \operatorname{Hom}_{R}\left(\widehat{R}^{\mathfrak{a}}, M\right) \rightarrow \mathrm{C}_{M}^{\mathfrak{a}}$ are inverse isomorphisms. In particular, $\operatorname{Hom}_{R}\left(\widehat{R}^{\mathfrak{a}}, M\right)$ is finitely generated over $R$.

Proof. One checks from the definitions that the composition $k_{M}^{\mathfrak{a}} f_{M}^{\mathfrak{a}}$ is the identity on $\mathrm{C}_{M}^{\mathfrak{a}}$. Hence, the first conclusion will be verified once we show that $k_{M}^{\mathfrak{a}}$ is bijective; the second conclusion will then follow, as $\mathrm{C}_{M}^{\mathfrak{a}}$ is finitely generated over $R$.

The module $\mathrm{C}_{M}^{\mathfrak{a}}$ is a-adically complete, so Proposition 2.4 implies that the evaluation map $g_{C_{M}^{\mathfrak{a}}}^{\mathfrak{a}}: \operatorname{Hom}_{R}\left(\widehat{R}^{\mathfrak{a}}, \mathrm{C}_{M}^{\mathfrak{a}}\right) \rightarrow \mathrm{C}_{M}^{\mathfrak{a}}$ is bijective. By Lemma 1.3 the map $\operatorname{Hom}_{R}\left(\widehat{R}^{\mathfrak{a}}, i_{M}^{\mathfrak{a}}\right): \operatorname{Hom}_{R}\left(\widehat{R}^{\mathfrak{a}}, \mathrm{C}_{M}^{\mathfrak{a}}\right) \rightarrow \operatorname{Hom}_{R}\left(\widehat{R}^{\mathfrak{a}}, M\right)$ is an isomorphism. In particular, the composition $k_{M}^{\mathfrak{a}}=g_{C_{M}^{\mathfrak{a}}}^{\mathfrak{a}} \circ \operatorname{Hom}_{R}\left(\widehat{R}^{\mathfrak{a}}, i_{M}^{\mathfrak{a}}\right)^{-1}$ is bijective, as desired.

\section{Detecting Completeness}

3.1. Proof of Theorem A. The implication (ii) $\Longrightarrow$ (iii) follows from Proposition 2.4 and Remark 2.5, and (iii) $\Longrightarrow$ (iii) is trivial.

For the implication (iii) $\Longrightarrow$ (ii), set $S=R / \operatorname{Ann}_{R}(M)$. A result of Gruson and Raynaud [15, Seconde Partie, Thm. (3.2.6)], and Jensen [12, Prop. 6] provides the following bound on the projective dimension of $\widehat{S}^{\mathfrak{a}}$ as an $S$-module:

$$
\operatorname{pd}_{S}\left(\widehat{S}^{\mathfrak{a}}\right) \leqslant \operatorname{dim}(S)=\operatorname{dim}_{R}(M) .
$$

Consider the following sequence of isomorphisms in $\mathrm{D}(R)$ :

$$
\begin{aligned}
\operatorname{RHom}_{R}\left(\widehat{R}^{\mathfrak{a}}, M\right) & \simeq \mathbf{R} \operatorname{Hom}_{R}\left(\widehat{R}^{\mathfrak{a}}, \mathbf{R H o m}_{S}(S, M)\right) \\
& \simeq \mathbf{R H o m}_{S}\left(\widehat{R}^{\mathfrak{a}} \otimes \mathbf{L} S, M\right) \\
& \simeq \mathbf{R H o m}_{S}\left(\widehat{S}^{\mathfrak{a}}, M\right) .
\end{aligned}
$$

The first isomorphism follows from the fact that $M$ is naturally an $S$-module. The second is adjunction, and the third is standard as $S$ is finitely generated over $R$. Combining (囷) with the displayed isomorphisms, the assumption $\operatorname{Ext}_{R}^{i}\left(\widehat{R}^{\mathfrak{a}}, M\right)=0$ for all $i=1, \ldots, \operatorname{dim}_{R}(M)$ implies $\operatorname{Ext}_{R}^{i}\left(\widehat{R}^{\mathfrak{a}}, M\right)=0$ for all $i \neq 0$. 
It follows that the natural map $\lambda: \operatorname{Hom}_{R}\left(\widehat{R}^{\mathfrak{a}}, M\right) \rightarrow \mathbf{R} \operatorname{Hom}_{R}\left(\widehat{R}^{\mathfrak{a}}, M\right)$ is an isomorphism in $\mathrm{D}(R)$. Proposition [2.6 implies that the composition $\lambda \circ f_{M}^{\mathfrak{a}}: \mathrm{C}_{M}^{\mathfrak{a}} \rightarrow$ $\mathbf{R H o m}_{R}\left(\widehat{R}^{\mathfrak{a}}, M\right)$ is also an isomorphism in $\mathrm{D}(R)$. Because $M$ is finitely generated, the natural morphism $\mu: \mathbf{L} \Lambda^{\mathfrak{a}}(M) \rightarrow \widehat{M}^{\mathfrak{a}}$ is also an isomorphism in $\mathrm{D}(R)$. These data yield the following commutative diagram.

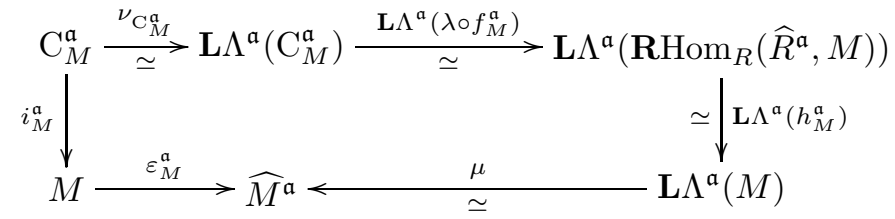

One sees that the composition of natural maps $\mathrm{C}_{M}^{\mathfrak{a}} \stackrel{i_{M}^{\mathfrak{a}}}{\longrightarrow} M \stackrel{\varepsilon_{M}^{\mathfrak{a}}}{\longrightarrow} \widehat{M}^{\mathfrak{a}}$ is bijective. Since $\varepsilon_{M}^{\mathfrak{a}}$ is also injective, the result now follows.

Remark 3.2. As the referee indicated, one can interpret Theorem $\AA$ as a statement about the $\mathfrak{a}$-adic completeness of $R / \operatorname{Ann}_{R}(M)$ because $M$ is $\mathfrak{a}$-adically complete if and only if $R / \operatorname{Ann}_{R}(M)$ is a-adically complete. For the sake of completeness, we include a sketch of the proof.

For one implication, assume that $M$ is a-adically complete. For each prime $\mathfrak{p} \in \operatorname{Ass}_{R}(M)$, the injection $R / \mathfrak{p} \hookrightarrow M$ and the completeness of $M$ imply that $R / \mathfrak{p}$ is a-adically complete. In particular, this is true for each minimal prime $\mathfrak{p}$ containing $\operatorname{Ann}_{R}(M)$, and it follows that the same is true for each nonminimal prime $\mathfrak{p}$ containing $\operatorname{Ann}_{R}(M)$. A prime filtration argument applied to $R / \operatorname{Ann}_{R}(M)$ shows that $R / \operatorname{Ann}_{R}(M)$ is a-adically complete.

Conversely, if $R / \operatorname{Ann}_{R}(M)$ is a-adically complete, then there exists an integer $r$ and a surjection $\left(R / \operatorname{Ann}_{R}(M)\right)^{r} \rightarrow M$, and it follows that $M$ is a-adically complete.

From this fact, one easily deduces the following: When $N$ is a second finitely generated $R$-module, if $M$ is a-adically complete and $\operatorname{Supp}_{R}(N) \subseteq \operatorname{Supp}_{R}(M)$, then $N$ is a-adically complete.

3.3. Proof of Theorem B. One implication is trivial. For the other, assume that $\widehat{R}^{\mathfrak{a}}$ is module-finite over $R$. As $\widehat{R}^{\mathfrak{a}}$ is flat and module-finite over $R$, it is projective, and so $\operatorname{Ext}_{R}^{i}\left(\widehat{R}^{\mathfrak{a}}, R\right)=0$ for each $i \neq 0$. The completeness of $R$ follows from Theorem $\mathrm{A}$.

The next example shows that the nontrivial implication in Corollary B fails if $\mathfrak{a}$ is not assumed to be in the Jacobson radical of $R$.

Example 3.4. Let $k$ be a field and set $R=k \times k$ and $\mathfrak{b}=k \times 0$. The Jacobson radical of $R$ is 0 . One readily checks that $\widehat{R}^{\mathfrak{b}} \cong 0 \times k$, showing that $R$ is not $\mathfrak{b}$-adically complete even though $\widehat{R}^{\mathfrak{b}}$ is module-finite over $R$.

Theorem $\mathrm{A}$ provides the converse to [3, (2.3)] when $R$ is local and $M$ is finitely generated. This is the implication (iii) $\Longrightarrow$ (ii) in the next result. The implication (ii) $\Longrightarrow$ (iii) is in [8, (3.7)] or [3, (2.3)], while the implication (iii) $\Longrightarrow$ (iii) is trivial.

Corollary 3.5. Let $(R, \mathfrak{m})$ be a local ring. For a finitely generated $R$-module $M$ the following conditions are equivalent:

(i) $M$ is $\mathfrak{m}$-adically complete.

(ii) For each flat $R$-module $B$ and each $i \neq 0$, one has $\operatorname{Ext}_{R}^{i}(B, M)=0$. 
(iii) For each $i \neq 0$, one has $\operatorname{Ext}_{R}^{i}\left(\widehat{R}^{\mathfrak{m}}, M\right)=0$.

With Theorem $\mathrm{A}$ and Corollary 3.5 in mind, one may ask what the finitely generated complete $R$-modules look like, say, when $R$ is not complete. Examples include the modules of finite length. We observe next that one can have complete $R$-modules of infinite length.

Example 3.6. Let $(S, \mathfrak{n})$ be a non-Artinian complete local ring. Set $R=S[X]_{(\mathfrak{n}, X)}$ with maximal ideal $\mathfrak{m}=(\mathfrak{n}, X) R$. The ring $R$ is not $\mathfrak{m}$-adically complete, while the module $R /(X) R \cong S$ is $\mathfrak{m}$-adically complete and has infinite length.

A finitely generated $R$-module $C$ is semidualizing if $R \stackrel{\simeq}{\longrightarrow} \mathbf{R H o m}_{R}(C, C)$.

Corollary 3.7. If $C$ is a semidualizing $R$-module such that $\operatorname{Ext}_{R}^{i}\left(\widehat{R}^{\mathfrak{a}}, C\right)=0$ for all $i \neq 0$, then $R$ is a-adically complete.

Proof. Theorem $\AA$ implies that $C$ is a-adically complete and hence $C \simeq C \otimes_{R} \widehat{R}^{\mathfrak{a}} \simeq$ $C \otimes_{R}^{\mathbf{L}} \widehat{R}^{\mathfrak{a}}$. By [5, (5.8)] the complex $C \otimes_{R}^{\mathbf{L}} \widehat{R}^{\mathfrak{a}}$ is $\widehat{R}^{\mathfrak{a}}$-semidualizing. This provides (1) in the following sequence while (4) and (5) are by hypothesis:

$$
\begin{aligned}
\widehat{R}^{\mathfrak{a}} & \stackrel{(1)}{\simeq} \mathbf{R H o m}_{\widehat{R}^{\mathfrak{a}}}\left(C \otimes_{R}^{\mathbf{L}} \widehat{R}^{\mathfrak{a}}, C \otimes_{R}^{\mathbf{L}} \widehat{R}^{\mathfrak{a}}\right) \\
& \stackrel{(2)}{\simeq} \mathbf{R} \operatorname{Hom}_{R}\left(C, \mathbf{R} \operatorname{Hom}_{\widehat{R}^{\mathfrak{a}}}\left(\widehat{R}^{\mathfrak{a}}, C \otimes_{R}^{\mathbf{L}} \widehat{R}^{\mathfrak{a}}\right)\right. \\
& \stackrel{(3)}{\simeq} \mathbf{R} \operatorname{Hom}_{R}\left(C, C \otimes_{R}^{\mathbf{L}} \widehat{R}^{\mathfrak{a}}\right) \\
& \stackrel{(4)}{\simeq} \mathbf{R} \operatorname{Hom}_{R}(C, C) \\
& \stackrel{(5)}{\simeq} R .
\end{aligned}
$$

(2) is adjunction [5, (1.5.2)] and (3) is standard [5, (1.5.5)].

Here is a version of Theorem $\mathrm{A}$ for complexes.

Proposition 3.8. Let $M$ be a homologically degreewise finite $R$-complex such that $\inf \left(\mathbf{L} \Lambda^{\mathfrak{a}}(M)\right)=\inf (M)$ and $\sup \left(\mathbf{L} \Lambda^{\mathfrak{a}}(M)\right)=\sup (M)$, e.g., if $M$ is homologically finite. Fix an integer $s \geqslant \sup (M)$. If $\mathbf{R} \operatorname{Hom}_{R}\left(\widehat{R}^{\mathfrak{a}}, M\right)$ is homologically concentrated in degree $s$, then so is $M$, and the module $\mathrm{H}_{s}(M)$ is $\mathfrak{a}$-adically complete.

Proof. Assume $M \neq 0$. Then $\sup \left(\mathbf{L} \Lambda^{\mathfrak{a}}(M)\right)=\sup (M)>-\infty$, and Lemma 2.2 implies $\mathbf{R H o m}_{R}\left(\widehat{R}^{\mathfrak{a}}, M\right) \nsucceq 0$. Our hypotheses provide (1) and (3) in the sequence

$$
s \stackrel{(1)}{\geqslant} \sup (M) \stackrel{(2)}{\geqslant} \sup \left(\mathbf{R H o m} \operatorname{Hom}_{R}\left(\widehat{R}^{\mathfrak{a}}, M\right)\right) \stackrel{(3)}{=} s
$$

and (2) is from [7, (2.1)]; this implies $s=\sup (M)$. Since $\mathbf{R H o m}\left(\widehat{R}^{\mathfrak{a}}, M\right)$ is homologically concentrated in degree $s$, one has $\Sigma^{s} \operatorname{Ext}_{R}^{-s}\left(\widehat{R}^{\mathfrak{a}}, M\right) \simeq \mathbf{R H o m}_{R}\left(\widehat{R}^{\mathfrak{a}}, M\right)$, providing the first of the following isomorphisms:

$$
\mathbf{L} \Lambda^{\mathfrak{a}}\left(\Sigma^{s} \operatorname{Ext}_{R}^{-s}\left(\widehat{R}^{\mathfrak{a}}, M\right)\right) \simeq \mathbf{L} \Lambda^{\mathfrak{a}}\left(\mathbf{R} \operatorname{Hom}_{R}\left(\widehat{R}^{\mathfrak{a}}, M\right)\right) \simeq \mathbf{L} \Lambda^{\mathfrak{a}}(M),
$$

while the second one is from Lemma 2.2. This provides (5) in the following sequence:

$$
\inf (M) \stackrel{(4)}{=} \inf \left(\mathbf{L} \Lambda^{\mathfrak{a}}(M)\right) \stackrel{(5)}{=} \inf \left(\mathbf{L} \Lambda^{\mathfrak{a}}\left(\Sigma^{s} \operatorname{Ext}_{R}^{-s}\left(\widehat{R}^{\mathfrak{a}}, M\right)\right)\right) \stackrel{(6)}{\geqslant} s \stackrel{(7)}{=} \sup (M) \stackrel{(8)}{\geqslant} \inf (M)
$$


while (4) is by assumption, (6) is by 2.1 (g), (7) is proved above, and (8) is trivial. It follows that $\inf (M)=\sup (M)=s$ and so $M$ is homologically concentrated in degree $s$. Finally, one has $M \simeq \Sigma^{s} \mathrm{H}_{s}(M)$ and so

$$
\mathbf{R H o m}_{R}\left(\widehat{R}^{\mathfrak{a}}, M\right) \simeq \mathbf{R H o m}_{R}\left(\widehat{R}^{\mathfrak{a}}, \Sigma^{s} \mathrm{H}_{s}(M)\right) \simeq \Sigma^{s} \mathbf{R} \operatorname{Hom}_{R}\left(\widehat{R}^{\mathfrak{a}}, \mathrm{H}_{s}(M)\right) .
$$

Since this is homologically concentrated in degree $s$, one has $\operatorname{Ext}_{i}^{R}\left(\widehat{R}^{\mathfrak{a}}, \mathrm{H}_{s}(M)\right)=0$ for each $i \neq 0$. Theorem $\mathrm{A}$ implies that $\mathrm{H}_{s}(M)$ is a-adically complete.

The next result contains Theorem C from the Introduction.

Corollary 3.9. Let $M, N$ be homologically finite $R$-complexes and $s \in \mathbb{Z}$ such that $s \geqslant \sup \left(\mathbf{R H o m}_{R}(N, M)\right)$. If $\mathbf{R} \operatorname{Hom}_{R}\left(\widehat{N}^{\mathfrak{a}}, M\right)$ is homologically concentrated in degree $s$, then so is $\mathbf{R H o m}_{R}(N, M)$, and $\operatorname{Ext}_{R}^{-s}(N, M)$ is a-adically complete.

Proof. 2.1(还) and adjunction provide the following sequence:

$$
\mathbf{R H o m}_{R}\left(\widehat{N}^{\mathfrak{a}}, M\right) \simeq \mathbf{R H o m}_{R}\left(\widehat{R}^{\mathfrak{a}} \otimes_{R}^{\mathbf{L}} N, M\right) \simeq \mathbf{R H o m}_{R}\left(\widehat{R}^{\mathfrak{a}}, \mathbf{R H o m}_{R}(N, M)\right) .
$$

Lemma 2.3 shows that Proposition 3.8 applies to the $\operatorname{complex} \operatorname{RHom}_{R}(N, M)$, yielding the desired conclusion.

Corollary 3.10. Assume that $R$ is local and $M, N$ are nonzero finitely generated $R$-modules with $\operatorname{pd}_{R}(N)<\infty$. If $\operatorname{Ext}_{R}^{i}\left(\widehat{N}^{\mathfrak{a}}, M\right)=0$ for each $i \neq 0$, then $N$ is free and $M$ is a-adically complete.

Proof. Using $s=0$ in Corollary 3.9, one concludes that $\operatorname{Ext}_{R}^{i}(N, M)=0$ for each $i \neq 0$ and that $\operatorname{Hom}_{R}(N, M)$ is a-adically complete. Since $\operatorname{pd}_{R}(N)$ is finite, one has

$$
\mathbf{R H o m}_{R}(N, M) \simeq \mathbf{R H o m}_{R}(N, R) \otimes_{R}^{\mathbf{L}} M
$$

by tensor-evaluation [2, (4.4)]. The next equalities are from [7, (2.1)] and [5, (2.13)]:

$$
0=\inf \left(\mathbf{R H o m}_{R}(N, M)\right)=\inf \left(\mathbf{R H o m}_{R}(N, R)\right)+\inf (M)=-\operatorname{pd}_{R}(N) .
$$

Since $R$ is local, the module $N \neq 0$ is free and $\operatorname{Hom}_{R}(N, M) \cong M^{n}$ for some $n>0$. Because $M^{n}$ is a-adically complete, the same is true of $M$.

\section{ACKNOWLEDGMENTS}

We are grateful to Phillip Griffith, Srikanth Iyengar, Christian U. Jensen, and Anders Thorup for stimulating discussions about this research. We also thank the anonymous referee for helpful comments.

\section{REFERENCES}

1. L. Alonso Tarrío, A. Jeremías López, and J. Lipman, Local homology and cohomology on schemes, Ann. Sci. École Norm. Sup. (4) 30 (1997), no. 1, 1-39. MR1422312 (98d:14028)

2. L. L. Avramov and H.-B. Foxby, Homological dimensions of unbounded complexes, J. Pure Appl. Algebra 71 (1991), 129-155. MR.93g:18017

3. R.-O. Buchweitz and H. Flenner, Power series rings and projectivity, Manuscripta Math. 119 (2006), no. 1, 107-114. MR2194381 (2007a:13025)

4. L. W. Christensen, Gorenstein dimensions, Lecture Notes in Mathematics, vol. 1747, SpringerVerlag, Berlin, 2000. MR2002e:13032

5. $ـ$ Semi-dualizing complexes and their Auslander categories, Trans. Amer. Math. Soc. 353 (2001), no. 5, 1839-1883. MR2002a:13017

6. L. W. Christensen, A. Frankild, and H. Holm, On Gorenstein projective, injective and flat dimensions - a functorial description with applications, J. Algebra 302 (2006), no. 1, 231-279. MR 2236602 
7. H.-B. Foxby, Isomorphisms between complexes with applications to the homological theory of modules, Math. Scand. 40 (1977), no. 1, 5-19. MR0447269 (56:5584)

8. A. Frankild, Vanishing of local homology, Math. Z. 244 (2003), no. 3, 615-630. MR 1992028 (2004d:13027)

9. S. I. Gelfand and Y. I. Manin, Methods of homological algebra, Springer-Verlag, Berlin, 1996. MR.1438306 (97j:18001)

10. J. P. C. Greenlees and J. P. May, Derived functors of I-adic completion and local homology, J. Algebra 149 (1992), no. 2, 438-453. MR1172439 (93h:13009)

11. R. Hartshorne, Residues and duality, Lecture Notes in Mathematics, vol. 20, Springer-Verlag, Berlin, 1966. MR 36:5145

12. C. U. Jensen, On the vanishing of $\lim ^{(i)}$, J. Algebra 15 (1970), 151-166. MR0260839(41:5460)

13. L Les foncteurs dérivés de $\varliminf_{\longleftarrow}$ et leurs applications en théorie des modules, Lecture Notes in Mathematics, vol. 254, Springer-Verlag, Berlin, 1972. MR0407091 (53:10874)

14. J. Lipman, Lectures on local cohomology and duality, Local cohomology and its applications (Guanajuato, 1999), Lecture Notes in Pure and Appl. Math., vol. 226, Dekker, New York, 2002, pp. 39-89. MR1888195 (2003b:13027)

15. M. Raynaud and L. Gruson, Critères de platitude et de projectivité. Techniques de "platification" d'un module, Invent. Math. 13 (1971), 1-89. MR0308104 (46:7219)

16. N. Spaltenstein, Resolutions of unbounded complexes, Compositio Math. 65 (1988), no. 2, 121-154. MR932640 (89m:18013)

Department of Mathematics, Institute for Mathematical Sciences, University of Copenhagen, Universitetsparken 5, 2100 København, Denmark

Department of Mathematics, California State University, Dominguez Hills, 1000 E. Victoria Street, Carson, California 90747

Current address: Department of Mathematics, North Dakota State University, 300 Minard

Hall, Fargo, North Dakota 58105-5075

E-mail address: sean.sather-wagstaff@ndsu.edu

$U R L:$ http://www.math.ndsu.nodak.edu/faculty/ssatherw 